\title{
Human Skill Performance to Control an Underactuated Pendulum-Driven Capsule System
}

\author{
Keattikorn Samarnggoon ${ }^{1}$, Shuang Cang ${ }^{2}$, Hongnian $\mathrm{Yu}^{3}$, Mohammad S. Hasan ${ }^{1}$, Tobias Flämig ${ }^{4}$
}

\begin{abstract}
${ }^{1}$ Faculty of Computing, Engineering and Science, Staffordshire University, Beaconside, Stafford ST18 0AD, UK
${ }^{2}$ School of Tourism, Bournemouth University, Fern Barrow, Poole, Dorset BH12 5BB, UK

${ }^{3}$ Faculty of Science and Technology, Bournemouth University, Fern Barrow, Poole, Dorset BH12 5BB, UK

${ }^{4}$ Baden-Wuerttemberg Cooperative State University, 70174 Stuttgart, Germany
\end{abstract}

\begin{abstract}
This paper investigates human learning and skill performance to control an underactuated pendulum-driven capsule system within an interactive virtual simulation environment. A number of experiments is conducted with 9 participants who learned to control the robot using a physical joystick. The results show differences in learning and skill performance among the participants. Right-handed and lefthanded participants achieved their highest trial on the opposite side of their handedness. High learning participant tends to achieve high performance whereas participant who has steady learning tends to produce stable performance either low or high. The variance of the displacements achieved appears to be a learning indicator while the high frequency of joystick oscillation at the right portion and interval gives high performance results.
\end{abstract}

Keywords-human factor; human adaptive mechatronics; virtual simulation; human skill; human learning

\section{INTRODUCTION}

Although advances in technology have evolved a machine to become more autonomous/automated, many machines still require human operators to operate and interact with them either fully manual, semi-manual, or supervisory controls especially in human centred machine such as lower limbs walking support structure for elderly or disabled, prosthesis, wheelchair etc. As a consequence, human control behaviour and performance have become the main focus in human adaptive mechatronics (HAM) research.

A HAM concept aims to improve a machine with the capability to adjust itself based upon the performance level of the human user [1], [2], [3], [4], [5]. The main idea behind HAM comes from that humans can learn to operate machines. In contrast, it is interesting to develop a machine that could learn to provide assistance to its user based on an individual skill performance. To achieve this type of machine, several methods and techniques such as human behaviour study, pattern recognition of the human operator actions, human skill performance evaluation during the machine operation, the interaction model between human and machine, the machine system modelling are being studied.

Human machine control performance and evaluation are the important HAM components because they provide a basis for the machine to give the appropriate adjustment and assistant. Without the knowledge of human operation performance, the machine would have no information for the adaptive adjustment or assistant.

In this paper we investigate human learning and performance to control an underactuated pendulum-driven capsule system within an interactive virtual simulation environment.

The paper is organised into the following sections. A review of the related works is presented in Section II. The proposed human interaction model appears in Section III. The experimentation procedures and settings are presented in Section IV. The results from the experiments are summarised in Section V. The discussion and conclusion are presented in Section VI and VII, respectively.

\section{RELATED WORKS}

Human skills have been long studied in a number of classical research fields e.g. psychology, sports, human factor engineering etc. [6], [7], [8]. However, there exist few studies on the human skill performance evaluation when operating a machine according to the HAM concept.

In a research area called haptic shared control (HSC) several studies have focused on human sharing control simultaneously with the machine. A special haptic force feedback device has been employed to help assist human driver in a car lane keeping task which proven to reduce the control activity by $16 \%$ [9], [10]. A performance and training enhancement by applying HSC to give a virtual force field a.k.a. 'virtual fixture' has proven to improve the performance. However, for a training enhancement it is ineffective because the operator tend to rely on the existence of the shared assistance [11].

Rasmussen divided the human performance behaviour into three levels i.e. skill, rule, and knowledge [12]. At the skill level behaviour, manipulations by humans are merely based upon voluntary movements, which behave like an automated action without consciousness. Those actions are extremely integrated, smooth, and can hardly be decomposed into elements without careful attention. This human low-level skill phenomenon is also confirmed in the study of professional musicians such as violinists and cellists who are hardly able to specifically describe the components to their performance [13]. At the rule level behaviour, the human use the stored rules, know-how, or instruction to control their actions. In other words, control is ruled by past successful experiences either 
from personal or vicarious/indirect experiences. At the highest level, knowledge level behaviour usually occurs in unfamiliar situations because previous experiences, rules, or know-how cannot be applied directly. Critical thinking, problem solving strategy and a modified plan of actions are tested against the desired goal heuristically.

In [14], four approaches to human performance modelling; information processing, control theory, task network, and knowledge based are studied for a number of useful applications e.g. in system design, system development, and system evaluation. Having a human performance model has benefits such as reducing risk, cost, and danger prior to the actual system implementation.

A brain monitoring system to investigate voluntary motion is studied to reveal the relation between brain activation area and skilled motion [15]. A near-infrared spectroscopy (NIRS) brain monitoring technique is used while the participants are asked to perform a drawing task by looking at the mirror instead of looking at the paper directly. The proposed index is used to evaluate and classify the skill levels into three categories high-skilled (HS), middle-skilled (MS), and lowskilled (LS). High-skilled persons show that there is activation in a premotor cortex (PMC) and supplementary motor area (SMA) during the early phase of the task performance which decreases gradually later on as an indicator of normal skilled action.

Discrete operator's hands movement during machine console operation have been investigated to evaluate the operator performance in [16], [17]. Fitts' law [18] is applied and validated in the study for the task which has partly visual feedback such as machine console operation. The machine console operation is considered as partly visual feedback because it does not intensively require perception through the eye. The outcome indicates that it is possible to use discrete hand motion to estimate skill level of the machine operator. In other words, there is a difference in the sequences of hand movement among novice and expert operators. A novice seems to be prone to unnecessary sequence of motions while the expert motions are optimised. In addition, eye gaze tracking [19] is applied to gather the operator eyes gaze while performing machine console operation.

A human performance index (HPI) is a method to evaluate human operator performance by scoring the chosen performance variables such as speed and accuracy variables and then applying multilayer weighting criteria to obtain the performance index value [20]. The method is validated using an on screen mouse target hitting task to measure a user's time taken to reach a circle target on the screen, an average time used across the number of trials, a cursor path accuracy, and an accuracy on the clicked target. The Fitts' law is also applied in this study to validate the speed-accuracy trade-off of the task.

The work presented in this paper follows the concept from [21] which provides an interactive virtual simulation environment to allow human interaction with the system based on the HAM principle. However, instead of a single human operator learning heuristically to control and identify the possibilities of angle profile pattern of the pendulum-driven capsule system, a number of participants participate in this work allow performance measurement analysis from their control trials. Also, the difference in learning and control strategies among the participants can be analysed.

\section{HUMAN OPERATOR AND CAPSULE SYSTEM}

In this section, a human operator interaction model with the capsule system is proposed and the details of the pendulumdriven capsule system are presented.

\section{A. Human-capsule system interaction model}

Fig. 1 shows the human-capsule system interaction model in this study. The model consists of 4 building blocks which pass the control information throughout system paths. The human operator is given the goal of the task to be performed. The internal processing of human brain processes the provided information and takes action via the joystick interface to control the pendulum-driven capsule system. The joystick actions then translate into the inverted pendulum angle. A proper rotation of the inverted pendulum will drive the capsule system towards desire direction. However, this rotation is the main control that the individual human operator has to learn. The appearance of pendulum orientation and capsule position on the display acts as the feedback information to the human operator eyes to be perceived and react. Then, it is returned to the human operator internal processing to process the information and make progress to the capsule position as required by the given task goal.

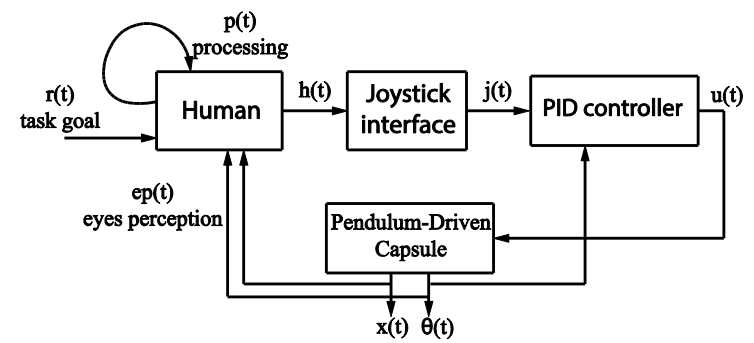

Figure 1 The human interaction model with the pendulum-driven capsule robot system.

Fig. 1 contains time-varying variables passing the information throughout the system paths. $r(t)$ is the reference or the given task goal, $\mathrm{p}(\mathrm{t})$ is an internal processing of an individual human brain, and $\mathrm{h}(\mathrm{t})$ is the result of an internal brain processing output as a hand motion to control the joystick interface. $j(t)$ is the output from the joystick which is generated by the human operator hand movement, $u(t)$ is the control output from the PID controller to the pendulum-driven capsule simulation according to the desired angle. $\theta(t)$ and $x(t)$ are outputs from the simulation model which appear on the screen of the virtual simulation platform and they act as feedback to the human operator visual perception. ep(t) is the simulation output information plus any external disturbances such as environmental distractions and unrelated activities on the screen.

\section{B. The pendulum-driven capsule model and simulation}

The schematic diagram of a pendulum-driven capsule model is shown on Fig. 2. The model is adopted from [22] with additional proportional-integral-differential (PID) controller applied to control the input torque to achieve the desired angle 
of the pendulum. In other words, the same mathematical model in [21] is applied with additional PID controller. The PID controller constants - $\mathrm{kP}, \mathrm{kI}, \mathrm{kD}$ are $0.7,0.7$, and 6.0, respectively. The control output gain factor is 10 . Table I shows the parameters for the pendulum-driven capsule system. Fig. 3 shows the 3D simulation of the model that is used for the experimentation.

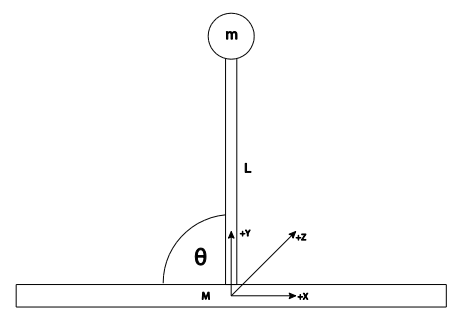

Figure 2 The pendulum-driven capsule system model.

TABLE I. THE CAPSULE SYSTEM PARAMETERS

\begin{tabular}{|c|c|c|c|c|}
\hline $\begin{array}{c}\text { Ball mass } \\
(\mathbf{k g})\end{array}$ & $\begin{array}{c}\text { Capsule } \\
\text { mass }(\mathbf{k g})\end{array}$ & $\begin{array}{c}\text { Shaft } \\
\text { length }(\mathbf{m})\end{array}$ & $\begin{array}{c}\text { Surface } \\
\text { friction } \\
\text { coefficient }\end{array}$ & $\begin{array}{c}\text { Gravity } \\
\text { constant } \\
\left(\mathbf{m} / \mathbf{s}^{\mathbf{2}}\right)\end{array}$ \\
\hline 0.2 & 0.5 & 0.3 & 0.5 & 9.81 \\
\hline
\end{tabular}

\section{THE EXPERIMENT}

The experiment is designed to investigate the human operator learning and skill performance when operating the pendulum-driven capsule system. This capsule system is chosen because it has a number of unique features [23], [24]. It is an unusual machine by its underactuated mechanism which is a good point in order to avoid benefit from past experience. A direct control of the angle of the pendulum requires hand motion skill to swing the pendulum at the right oscillation and timing to initiate the capsule robot to displace. As a consequence, it requires both learning and skill to operate this system.

As described in the interaction model, a joystick is used as the interaction interface for the human operator to operate the system. Fig. 4 shows the joystick and the corresponding axis used to control the angle of the pendulum directly. The direct angle control means that when the joystick is pushed towards negative $\mathrm{x}$ direction it will rotate the pendulum angle of the capsule system to left hand side which means toward the positive 90 degrees of the pendulum-driven capsule model (Fig. 2). A screenshot of the 3D simulation of the pendulumdriven capsule system is shown on Fig. 3. The sampling interval was at 10 milliseconds.

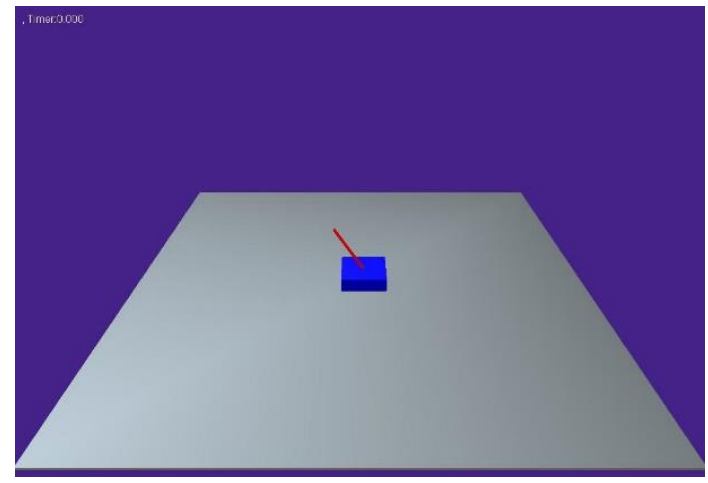

Figure 3 The screenshot of the simulation platform.

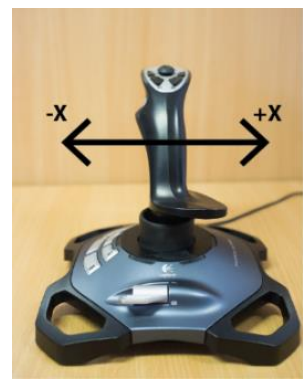

Figure 4 The joystick control interface and the axis uses to control the pendulum angle.

The given control task in this experiment is a direct pendulum angle control to displace the capsule to the specified direction i.e. left or right. A human controller has full control over the desired angle of the pendulum by pushing the joystick handle. The effect of pushing the handle will cause the capsule to move erratically back and forth. This is normal because of the mechanism of the inverted pendulum-driven capsule system. However, it is controllable for the intended displacement direction.

Each session of the experiment for each participant contained learning sessions prior to the actual performance trials. The actual trials consist of 6 trials separated into 3 trials for right and 3 trials for left movement. There is no time limitation for the learning session while the actual trial is limited to 20 seconds a session which means a participant has to control the capsule to the specified direction as far as possible within the provided time limit.

Nine participants ages between 21 and 50 attended this experiment. Each of them agreed and signed the consent form prior to the experiment session. The participants have different personal attributes such as ages, handedness, and knowledge about principles related to the machine which could influence the control learning and performance.

\section{RESULTS}

This section summarises the results from the experimentation conducted by nine participants. The naming conventions are described in this early of the section for clarification of the terms and abbreviations. The ' $\mathrm{P}$ ' letter followed by a number is used for participant identity. The ' $R$ ' and ' $\mathrm{L}$ ' letter indicate right or left movement task followed by a trial number in Figure 5. For examples, 'R1' stands for the first 


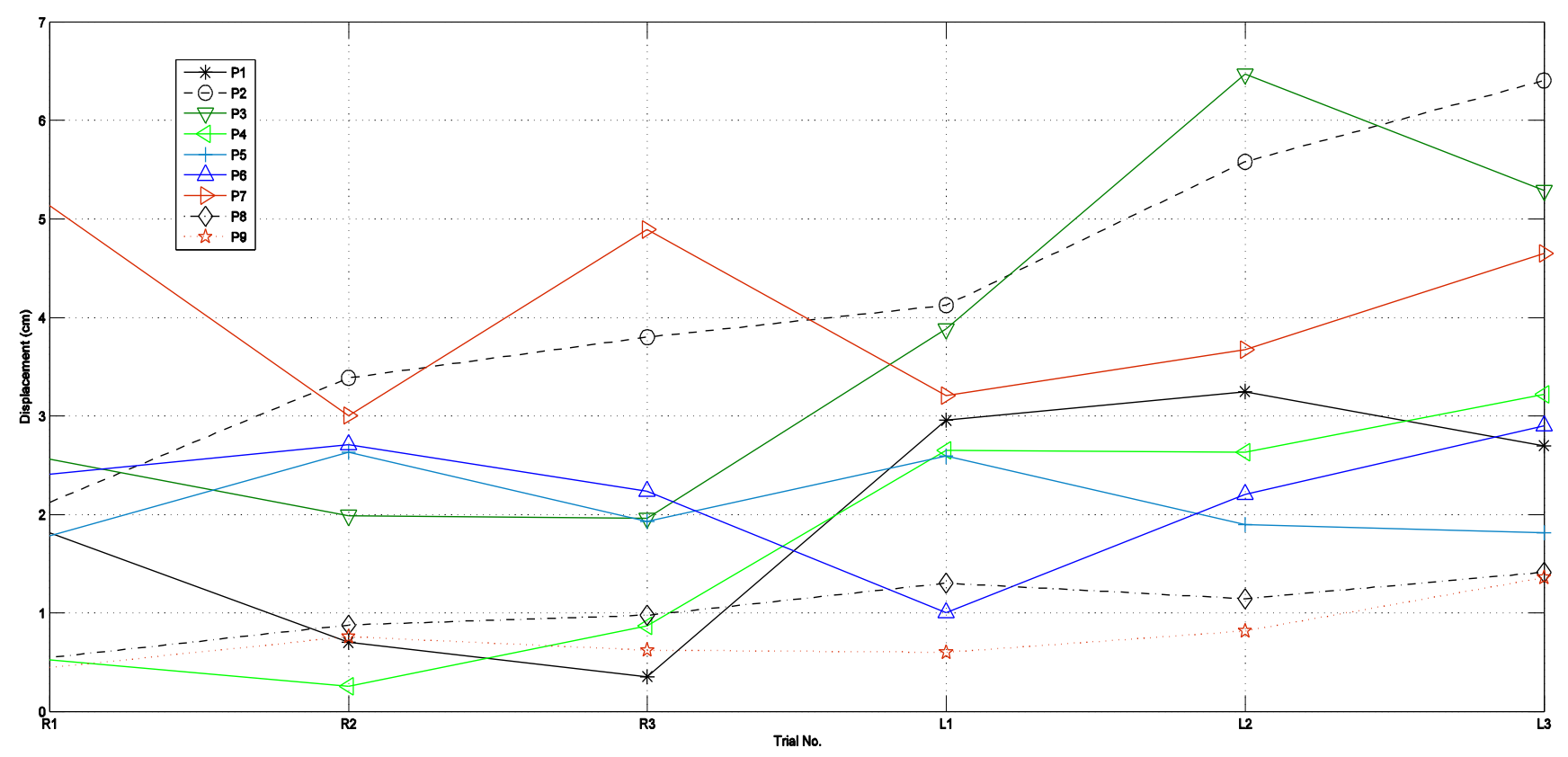

Figure 5 The absolute value of final capsule position.

right trial, 'R2' for the second right trial, and so on. Therefore, there are nine participant labelled from 'P1' to 'P9'. Each of the participant did perform the experiment for ' $\mathrm{R} 1$ ' to ' $\mathrm{R} 3$ ' and 'L1' to 'L3' produced a total of 54 trials for the entire experimentation from 9 participants.

The participant's attributes and knowledge regarding the theory related to the capsule system has been shown in Table II. Eight of them are male and seven of them are right handed while the other two are left handed for handedness. All of them know Newton's law of motion which is a basis to this capsule system model and almost all of them know about a pendulum. However, approximately half of them know an inverted pendulum and only three of them understand the principle on the inverted pendulum. None of the participants has tried this experimentation platform before.

TABLE II. THE PARTICIPANTS ATTRIBUTES AND KNOWLEDGE

\begin{tabular}{|c|c|c|c|c|c|c|c|c|}
\hline P & G & A & H & N & Pe & IPe & IpeP & TBF \\
\hline P1 & M & $31-35$ & Right & Y & Y & N & N & N \\
\hline P2 & M & $36-40$ & Right & Y & Y & Y & N & N \\
\hline P3 & F & $31-35$ & Right & Y & Y & N & N & N \\
\hline P4 & M & $21-25$ & Right & Y & N & N & N & N \\
\hline P5 & M & $26-30$ & Left & Y & Y & N & N & N \\
\hline P6 & M & $26-30$ & Right & Y & Y & Y & N & N \\
\hline P7 & M & $41-45$ & Left & Y & Y & Y & Y & N \\
\hline P8 & M & $46-50$ & Right & Y & Y & Y & Y & N \\
\hline P9 & M & $26-30$ & Right & Y & Y & Y & Y & N \\
\hline
\end{tabular}

$\mathrm{P}=$ Participant Identity, G=Gender, A=Ages, H=Handedness, N=Knowledge on Newton law of motion, $\mathrm{Pe}=$ Knowledge on pendulum, $\mathrm{IPe}=$ Knowledge on inverted pendulum, $\mathrm{IPeP}=$ Knowledge on inverted pendulum principle, $\mathrm{TBF}=\mathrm{Has}$ the participant tried this experiment before

TABLE III. LEARNING TIME, AVERAGE DISPLACEMENT, AND AVERAGE SPEED

\begin{tabular}{|c|c|c|c|c|c|c|c|}
\hline P & $\begin{array}{c}\text { LT } \\
(\text { sec })\end{array}$ & $\begin{array}{c}\text { Avg } \\
\text { R }\end{array}$ & $\begin{array}{c}\text { Avg } \\
\text { R }\end{array}$ & $\begin{array}{c}\text { Avg } \\
\text { L }\end{array}$ & $\begin{array}{c}\text { Avg L } \\
\text { Spd }\end{array}$ & $\begin{array}{c}\text { Tot. } \\
\text { ABS }\end{array}$ & $\begin{array}{c}\text { Tot. } \\
\text { Avg }\end{array}$ \\
\hline
\end{tabular}

\begin{tabular}{|l|r|c|c|c|c|c|c|}
\hline & & $\begin{array}{c}\text { Dis } \\
(\mathbf{c m})\end{array}$ & $\begin{array}{c}\text { Spd } \\
(\mathbf{c m} / \mathbf{s})\end{array}$ & $\begin{array}{c}\text { Dis } \\
(\mathbf{c m})\end{array}$ & $(\mathbf{c m} / \mathbf{s})$ & $\begin{array}{c}\text { Dis } \\
(\mathbf{c m})\end{array}$ & $\begin{array}{c}\text { Spd } \\
(\mathbf{c m} / \mathbf{s})\end{array}$ \\
\hline P1 & 78.79 & 0.89 & 0.045 & -2.90 & -0.145 & 11.38 & 0.95 \\
\hline P2 & 100.76 & 3.07 & 0.154 & -5.34 & -0.267 & 25.22 & 2.10 \\
\hline P3 & 212.9 & 2.12 & 0.106 & -5.15 & -0.257 & 21.79 & 1.82 \\
\hline P4 & 260.8 & 0.53 & 0.026 & -2.81 & -0.141 & 10.02 & 0.84 \\
\hline P5 & 141.97 & 2.07 & 0.104 & -2.06 & -0.103 & 12.39 & 1.03 \\
\hline P6 & 318.09 & 2.42 & 0.121 & -2.02 & -0.101 & 13.33 & 1.11 \\
\hline P7 & 98.88 & 4.31 & 0.216 & -3.82 & -0.191 & 24.41 & 2.03 \\
\hline P8 & 97.51 & 0.77 & 0.038 & -1.26 & -0.063 & 6.09 & 0.51 \\
\hline P9 & 586.88 & 0.60 & 0.030 & -0.90 & -0.045 & 4.50 & 0.38 \\
\hline
\end{tabular}

$\mathrm{P}=$ Participant identity, LT=Learning time, Avg R Dis=Average right displacement, Avg L Dis=Average left displacement, Avg R Spd=Average right speed, Avg L Spd=Average left speed, Tot. ABS Dis=Tota

Table III shows the results - learning time prior to the actual performance, average displacement achieved and average speed. A total absolute displacement and a total average speed are also calculated to see an overall performance of each participant. The absolute value of final capsule position for each of the trial for every participant is plotted and shown on Fig. 5.

\section{DISCUSSIONS}

In this section, interpretation of the results concerning learning and skill performance in the context of the capsule system control task is discussed.

According to Table III the amount of learning time (LT) does not reflect the performance of the actual trials. The best total absolute displacement achieved belongs to ' $\mathrm{P} 2$ ' who took 100.76s for learning time while 'P9' used 586.88 to learn to control the robot but the total absolute displacement achieved for 'P9' is the worst among all participants. This indicates that the participant 'P9' spent a large amount of time to figure out how the capsule system works but could not acquire sufficient skill to control the capsule system. 
The skill performance indicator is a final position of the capsule achieved within the time limitation of 20 seconds for a trial. As shown on Fig. 5 the highest performances belong to 'P2-L3' and 'P3-L2'. It is apparent that the best performer, P2, also exhibits the highest total average speed at $2.10 \mathrm{~cm} / \mathrm{s}$ (Tot. Avg Spd) as shown on Table III. 'P2' shows consistent performance. The lowest trial belong to 'P4-R2', however, the total average speed is on a moderate level at $0.84 \mathrm{~cm} / \mathrm{s}$.

Fig. 6 shows the average absolute speed for right and left control task. It is interesting that almost all of the right-handed participants gained more speed on the control task for the given left hand side movement task. Likewise, the left-handed participants, 'P5' and 'P7' achieved more speed on their right hand side tasks. This could be an effect of hand grasping orientation on the joystick control interface. This interesting fact conforms to performance achieved by the best trial which belongs to the left movement task by right handed participant 'P2-L3', while the maximum displacement achieved from left handed participants belongs to the right movement task 'P7R1'.

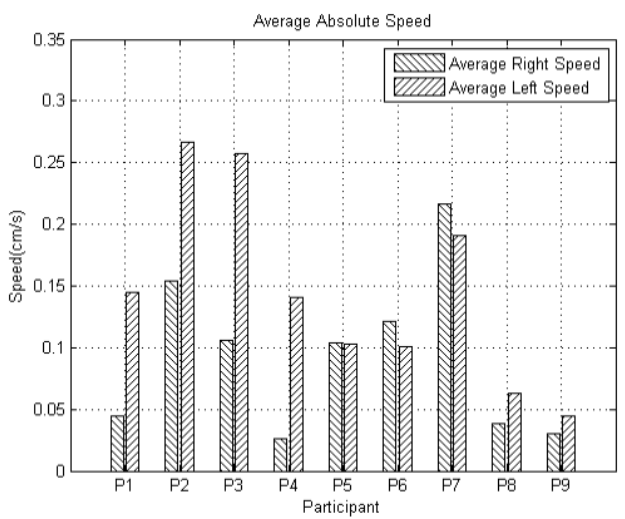

Figure 6. Graphs of the average absolute speed.

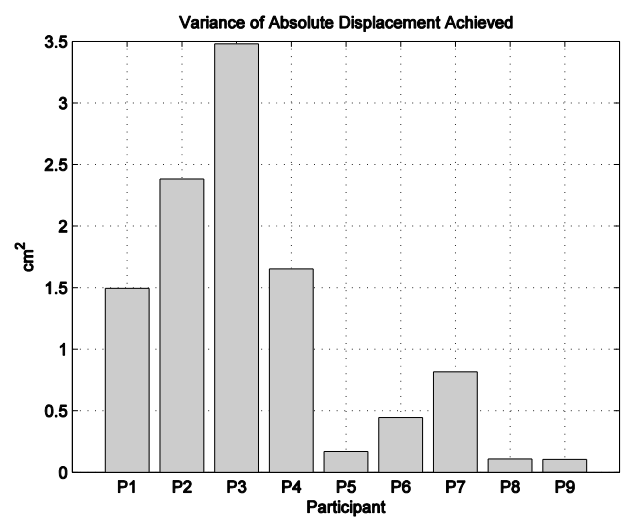

Figure 7. Variance of absolute displacement for each participant.

The variance of the absolute displacement achieved for each participant is calculated and is shown in Fig. 7. This value indicates consistency of performance for each of the participant across the performance trials. It could be used as a 'learning index'. For example, the participant ' $\mathrm{P} 3$ ' gained the highest learning performance because the first three right side trials (Fig. 5) are not very well but in the next three left trials the participant achieves almost as long displacement as the highest displacement achieved by 'P2'. This interpretation method is also applied to the participant 'P4' who gained a high learning performance indicator (variance). Although the participant 'P7' achieved good performance across all of the trials, the variance is relatively low. This means there was not much learning gained by this participant.

Fig. 8 shows the control input characteristics of two best and two worst trials from all of 54 trials. The two best trials from the participant 'P2-L3' and 'P3-L2' are shown on Fig. 8 (a) and Fig. 8 (b), respectively. The worst two performance trials are from participant 'P4-R2' and 'P1-R3' which are shown in Fig. 8 (c) and Fig. 8 (d), respectively.

The frequency characteristic of the control input from Fig. 8 shows clues for the high performance trials. The control inputs with high frequency i.e. around $1 \mathrm{~Hz}$ or more are from both of the highest trials, 'P2-L3' and 'P3-L2'. Also, this frequency is performed and maintained across the performance trials. In other words, it is performed at consistent frequency across the trials. In contrast, the low skill performer exhibits low frequency action, inconsistent across the trial. These control input characteristics were performed by 'P4-R2' and 'P1-R3'.

\section{CONCLUSIONS AND FUTURE WORKS}

Human learning and skill performance to control an underactuated pendulum-driven capsule system is studied in this paper. The human interaction model with the capsule system is proposed to explain the flow of control data and information throughout the system paths. The experimentation is carried out with 9 participants to study their learning and skill to control the capsule system. These participants exhibit different learning strategies, control strategies, and performance outcomes.

In this study, the learning time used prior to the actual performance does not reflect the actual trial sequences. However, the variance of the displacements achieved across all trials indicates the learning achievement. The final displacement of the capsule and the corresponding average speed are skill performance indicators. It appears that the participant who is able to oscillate the control input at high, consistent frequency, and at the appropriate portion on the joystick $\mathrm{x}$-axis has achieved relatively high performance.

Although the angle control pattern generated by human operator differs from the 6 steps control strategy in [22] the pattern successfully drives the capsule system forward, it is not a perfect trajectory as in automatic control. This is normal as a human being who can heuristically learn and apply knowledge at the facing circumstance to solve the problem but does not need to be mathematically/objectively perfect as in the automation.

In future works, an analysis of the time series of the control input will be studied to utilise the details of the participants hand control behaviour on the joystick interface, for example similarity, trend and seasonality of the control input. In addition, future experiment will include biometric measurement such as eye gaze, body movement, and surface brain activity during the participants control activity. These measurements will give a deeper understanding of the human learning, skill 

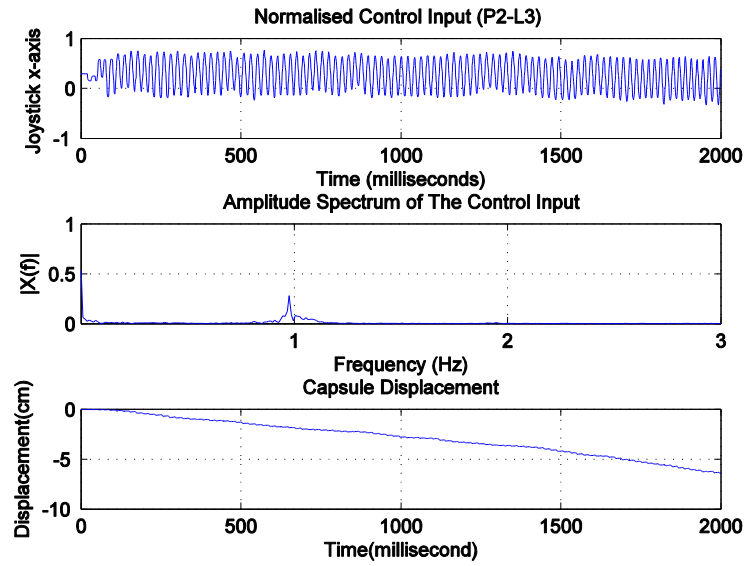

(a)
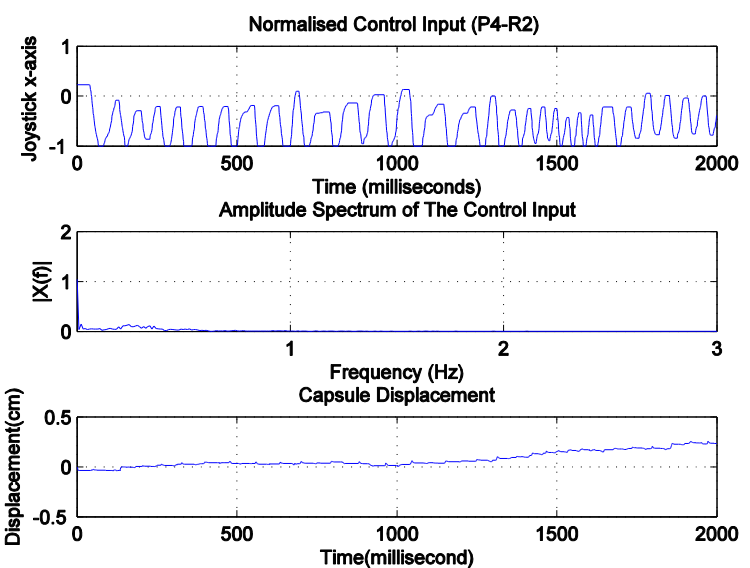

(c)
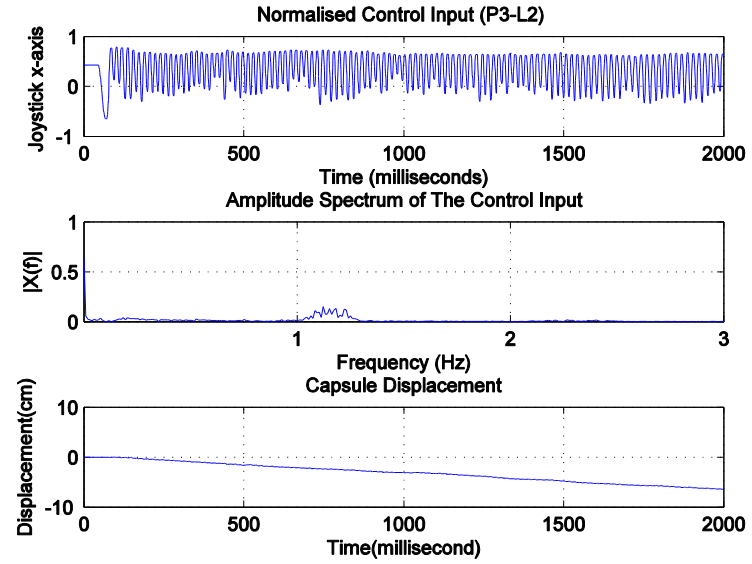

(b)
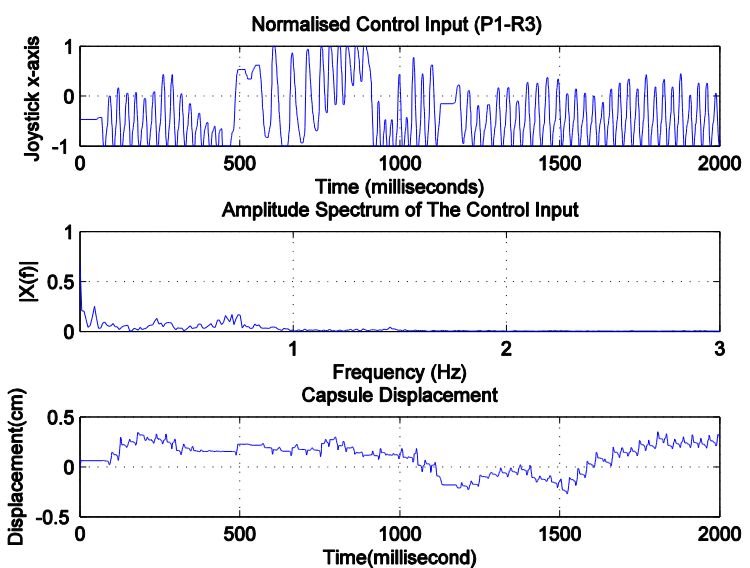

(d)

Figure 8 Graphs show normalised control input from the joystick, the frequency characteristics, and the capsule displacement.

performance, and control behaviour on the robot system control task under the HAM concept.

\section{ACKNOWLEDGMENT}

This work has been supported by the European ErasmusMundus Sustainable eTourism project 2010-2359, the EPSRC UK-Japan Network on Human Adaptive Mechatronics Project (EP/E025250/1) and EU Erasmus Mundus Project-ELINK (EM ECW-ref.149674-EM-1- 2008-1-UK-ERAMUNDUS).

\section{REFERENCES}

[1] Harashima, F. and Suzuki, S., "Human adaptive mechatronicsinteraction and intelligence," in Advanced Motion Control, 2006. 9th IEEE International Workshop on, 2006, pp. 1-8.

[2] S. Suzuki, "Human Adaptive Mechatronics," Industrial Electronics Magazine, IEEE, vol. 4, no. 2, pp. 28-35, 2010.

[3] S. Suzuki, H. Igarashi, H. Kobayashi, T. Yasuda, and F. Harashima, "Human Adaptive Mechatronics and Human-System Modelling," Int J Adv Robotic Sy, vol. 10, no. 152, 2013.

[4] H. Yu, "Guest Editorial," Proceedings of the Institution of Mechanical Engineers, Part I: Journal of Systems and Control Engineering, vol. 225, no. 6, pp. 705-708, 2011.
[5] H. Yu, "Editorial," International Journal of Modelling, Identification and Control, vol. 4, no. 4, pp. 299-303, 2008.

[6] G. Wulf, Attention and motor skill learning. Human Kinetics, 2007.

[7] M. D.Robb, The dynamics of motor-skill acquisition. Prentice Hall New Jersey, 1972.

[8] W. H. Edwards, Motor Learning and Control: From Theory to Practice. Cengage Learning, 2010.

[9] M. Mulder, D. A. Abbink, and E. R. Boer, "Sharing Control With Haptics: Seamless Driver Support From Manual to Automatic Control," Human Factors: The Journal of the Human Factors and Ergonomics Society, 2012.

[10] D. A. Abbink, M. Mulder, and E. R. Boer, "Haptic shared control: smoothly shifting control authority?," Cognition, Technology \& Work, pp. 1-10, 2012.

[11] M. K. O'Malley, A. Gupta, M. Gen, and Y. Li, "Shared control in haptic systems for performance enhancement and training," TRANSACTIONSAMERICAN SOCIETY OF MECHANICAL ENGINEERS JOURNAL OF DYNAMIC SYSTEMS MEASUREMENT AND CONTROL, vol. 128, no. 1 , p. $75,2006$.

[12] J. Rasmussen, "Skills, rules, and knowledge; signals, signs, and symbols, and other distinctions in human performance models.," Systems, Man and Cybernetics, IEEE Transactions on, vol. 13, no. 3, pp. 257-266, 1983.

[13] K. Furukawa, S. Igarashi, K. Ueno, T. Ozaki, S. Morita, N. Tamagawa, T. Okuyama, and I. Kobayashi, "Modeling human skill in bayesian 
network," Linkoping Electronic Articles in Computer and Information Science, vol. 7, p. 012, 2002.

[14] B. M. Huey, S. Baron, and D. S. Kruser, Quantitative modeling of human performance in complex, dynamic systems. National Academies Press, 1990.

[15] S. Suzuki, H. Kobayashi, and F. Harashima, "Brain monitoring analysis of skill on voluntary motion," in Control, Automation and Systems, 2007. ICCAS'07. International Conference on, 2007, pp. 1178-1182.

[16] S. Suzuki and F. Harashima, "Skill Evaluation from Observation of Discrete Hand Movements during Console Operation," Journal of Robotics, vol. 2010, pp. 1-13, 2010.

[17] S. Suzuki and F. Harashima, "Analysis of machine operation skills using hand discrete movement," in Emerging Technologies and Factory Automation, 2008. ETFA 2008. IEEE International Conference on, 2008, pp. 2824-2829.

[18] P. M. Fitts and M. I. Posner, "Human performance," 1967.

[19] J. Kolodko, S. Suzuki, and F. Harashima, "Eye-gaze tracking: an approach to pupil tracking targeted to FPGAs," in Intelligent Robots and
Systems, 2005.(IROS 2005). 2005 IEEE/RSJ International Conference on, 2005, pp. 344-349.

[20] T. Parthornratt, R. Parkin, and M. Jackson, "Human performance indexa generic performance indicator," Proceedings of the Institution of Mechanical Engineers, Part I: Journal of Systems and Control Engineering, vol. 225, no. 6, pp. 721-734, 2011.

[21] K. Samarnggoon and H. Yu, "Real time Virtual Simulation of an Underactuacted Pendulum-Driven Capsule System," in Control (CONTROL), 2012 UKACC International Conference on, Cardiff, 2012, vol. 2012 , pp. $568-573$

[22] H. Yu, Y. Liu, and T. Yang, "Closed-loop tracking control of a pendulum-driven cart-pole underactuated system," Proceedings of the Institution of Mechanical Engineers, Part I: Journal of Systems and Control Engineering, vol. 222, no. 2, pp. 109-125, 2008.

[23] Y. Liu and H. Yu, "A survey of underactuated mechanical systems," IET Control Theory and Applications, vol. 7, no. 7, pp. 1-15, Feb. 2013.

[24] M. W. Spong, "Underactuated mechanical systems," in Control Problems in Robotics and Automation, Springer, 1998, pp. 135-150. 